\title{
Impacts of Transportation Cost on Distribution-Free Newsboy Problems
}

\author{
Ming-Hung Shu, ${ }^{1}$ Chun-Wu Yeh, ${ }^{2}$ and Yen-Chen $\mathrm{Fu}^{3}$ \\ ${ }^{1}$ Department of Industrial Engineering \& Management, National Kaohsiung University of Applied Sciences, \\ 415 Chien Kung Road, Kaohsiung 80778, Taiwan \\ ${ }^{2}$ Department of Information Management, Kun Shan University, 195 Kunda Road, Yongkang District, Tainan 71003, Taiwan \\ ${ }^{3}$ Department of Industrial and Information Management, National Cheng Kung University, 1 University Road, Tainan 70101, Taiwan
}

Correspondence should be addressed to Yen-Chen Fu; r3897101@mail.ncku.edu.tw

Received 27 June 2014; Revised 3 September 2014; Accepted 13 September 2014; Published 30 September 2014

Academic Editor: Vikas Kumar

Copyright (C) 2014 Ming-Hung Shu et al. This is an open access article distributed under the Creative Commons Attribution License, which permits unrestricted use, distribution, and reproduction in any medium, provided the original work is properly cited.

A distribution-free newsboy problem (DFNP) has been launched for a vendor to decide a product's stock quantity in a singleperiod inventory system to sustain its least maximum-expected profits when combating fierce and diverse market circumstances. Nowadays, impacts of transportation cost on determination of optimal inventory quantity have become attentive, where its influence on the DFNP has not been fully investigated. By borrowing an economic theory from transportation disciplines, in this paper the DFNP is tackled in consideration of the transportation cost formulated as a function of shipping quantity and modeled as a nonlinear regression form from UPS's on-site shipping-rate data. An optimal solution of the order quantity is computed on the basis of Newton's approach to ameliorating its complexity of computation. As a result of comparative studies, lower bounds of the maximal expected profit of our proposed methodologies surpass those of existing work. Finally, we extend the analysis to several practical inventory cases including fixed ordering cost, random yield, and a multiproduct condition.

\section{Introduction}

A newsboy (newsvendor) problem has been initiated to determine the stock quantity of a product in a single-period inventory system when the product whose demand is stochastic has a single chance of procurement prior to the beginning of selling period. Aiming to maximize expected profit, decisive quantity trades off between the risk of underordering, which fails to gain more profit, and the loss of overordering, which compels release below the unit purchasing cost.

Traditional models for the newsboy problem assume that a single vendor encounters the demand of a product complying with a particular probability distribution function with known parameters, such as a normal, SchmeiserDeutsch, beta, gamma, or Weibull distribution [1]. With this assumption, several recent studies have to a certain extent succeeded in resolution of certain practical problems. For example, Chen and Ho [2] and Ding [3] analyzed the optimal inventory policy for newsboy problems with fuzzy demand and quantity discounts. Arshavskiy et al. [4] performed experimental studies by implementing the classical newsvendor problem in practice. Ozler et al. [5] studied a multiproduct newsboy problem under value-at-risk constraint; with loss-averse preferences, Wang [6] introduced a problem of multinewsvendors who compete with inventories setting from a risk-neutral supplier. When confronting myriad conditions in markets, however, in many occasions this designated distributional demand failed to best safeguard the vendor's profit.

To cope with the failure, models for the distributionfree newsboy problem (DFNP) have been broadly introduced over the past two decades. Gallego and Moon [7] first outlined a compacted analysis procedure for arranging optimal order quantities to certain inventory models, such as the single product, fixed ordering, random yield, and a multiproduct case. Alfares and Elmorra [8] further employed the procedure for the inventory model which considers shortage penalty cost. Moon and Choi [9] derived an ordering rule for the balking-inventory control model, where probability of per unit sold declines as inventory level falls below balking level. 
More recently, Cai et al. [10] provided measurements for deployment of multigenerational product development with the project cost accrued from different phases of a product life cycle such as development, service, and associated risks. Lee and Hsu [11] and Güler [12] developed an optimal ordering rule when an effect of advertising expenditure was reckoned on the inventory model. Kamburowski [13] presented new theoretical foundations for analyzing the best-case and worstcase scenarios. Due to prevalence of purchasing online, Mostard et al. [14] studied a resalable-return model for the distant selling retailers receiving internet orders from customers who have right to return their unfit merchandise in a stipulated period.

Over the past few years, energy prices have risen significantly and become more volatile; transportation of goods has become the highest operational expense as noted by Barry [15]. Many evidences indicate that in the US inbound freight costs for domestically sourced products and imported products typically range from 2 to $4 \%$ and from 6 to $12 \%$ of gross sales, respectively, and outbound transportation costs typically average $6 \%$ to $8 \%$ of net sales. In addition, Swenseth and Godfrey [16] reported that depending on the estimates utilized, upwards of $50 \%$ of the total annual logistic cost of a product could be attributed to transportation and that these costs were going up. UPS recently announced a $4.9 \%$ increase in its net average shipping rate. Ostensibly, the expenditure of the inbound/outbound material transportation has become a critical component of a total annual logistic cost function for determining purchase quantities. Effects of transportation have gained substantial recognition in vendor-buyer coordination problems.

Swenseth and Godfrey [16] unified two freight rate functions into a total annual cost function to understand their brunt on purchasing decisions. For integration of inventory and inbound/outbound transportation decisions, Çetinkaya and Lee [17] enabled an optimal inventory policy and Toptal et al. [18] carried out ideal cargo capacity and minimal costs. Toptal and Çetinkaya [19] further studied a coordination problem between a vendor and a buyer under explicit transportation consideration. More recently, Zhang et al. [20] generalized a standard newsboy model to the freight cost proportional to the number of the containers used. Toptal [21] studied exponentially/uniformly distributed demands and trucking costs. Mutlu and Çetinkaya [22] developed an optimal solution when inventory replenishment and shipment scheduling under common dispatch costs are considered.

Although impacts of the transportation cost on determination of the optimal inventory quantity have become attentive, its influence on the DFNP has not been fully investigated. To bridge the gap, this paper develops analytical and efficient procedures to acquire optimal policies for the DFNP in which the transportation cost function is explicitly joined into the vendor's expected profit structure. We borrowed the idea from the transportation management models [23] that the transportation cost is modeled as a function of delivery quantities; as a result of the computational studies, our proposed optimal-ordering rules increase lower bound of maximized expected profit as much as $4 \%$ on average, as opposed to the optimal policies recommended by Gallego and Moon [7].
Moreover, in order to determine and implement the optimal policies in practice, we perform comprehensive sensitivity analyses for the vital parameters, such as the demand mean and variance, unit cost of product, and transportation cost.

Lastly, this paper is organized as follows. Section 2 describes our model formulation for the DFNP in presence of transportation cost whose optimal order quantity $Q^{*}$ along with lower bound of maximized expected profit $E\left(Q^{*}\right)$ is resolved in Section 3. In Section 4, we study sensitivity analyses and comparative studies. A fixed-ordering cost case is analyzed in Section 5, while a random-yield case is considered in Section 6. In Section 7, we further contemplate a multiproduct case with budget constraint. Conclusions and Implications make up Section 8.

\section{Model Formulation for the DFNP with Transportation Cost}

For investigating impacts of the DFNP in consideration of the transportation cost, we briefly depict its model assumptions and notations used in this paper. Demand rate from a specific buyer is denoted by $D$, whose distribution $G$ is unknown with mean $\mu$ and variance $\sigma^{2}$. Note that the unknown distribution $G$ is equal to or better off the worst possible distribution $\vartheta$. With a product's unit cost $c$, a vendor orders size of $Q$, which arrive before delivering to the buyer. Intuitively, in one replenishment cycle, $\min \{Q, D\}$ units are sold with unit price $p$ and the unsold items $(Q-D)^{+}$are salable with unit salvage value $s$, where $s<p$, where $(Q-D)^{+}$defined as the positive part of $Q-D$ are equivalent to $\max \{Q-D, 0\}$. This implies $Q=\min \{Q, D\}+(Q-D)^{+}$.

Furthermore, we assume transportation cost is a function of the order quantity $Q$, denoted by $t c(Q)$. We further assume the transportation cost is in a general form of the tapering (or proportional) function; for example, $\operatorname{tc}(Q)=a+b \ln Q$, for $a, b \geq 0$, where $a$ and $b$ represent fixed and variable transportation cost. Intuitively, high volume corresponds to lower per unit rate of transportation, reflecting that the inequality $[\operatorname{tc}(Q) / Q]^{\prime} \leq 0$ holds true. That is, $[\operatorname{tc}(Q) / Q]^{\prime}=$ $(b-a-b \ln Q) / Q^{2} \leq 0$ or equivalently $Q \geq \exp (1-a / b)$, where the regulated minimal quantity level of delivery is $Q^{s}=$ $\exp (1-a / b)$ and $Q \geq Q^{s}$.

The assumption is based on the following observations from the existing works and UPS's on-site data set. First off, economic trade-off for the optimal transportation cost lies between provided service level and shipped quantity [17]. Secondly, in the shipment more weight signifies larger delivery quantity and higher shipment cost [19]. Thirdly, the transportation management models proposed by Swenseth and Godfrey [16] and Toptal et al. [18] indicated that optimal shipping quantity renders minimum of the transportation cost. Finally, we display the on-site shipping data set collected from the UPS worldwide expedited service at zone 7 shown in Figure 1.

Now, we are ready to combat the DFNP in presence of the transportation cost. Our purpose is to decide an optimal stock quantity in a single-period inventory system for a vendor to sustain its least maximum-expected profits when 


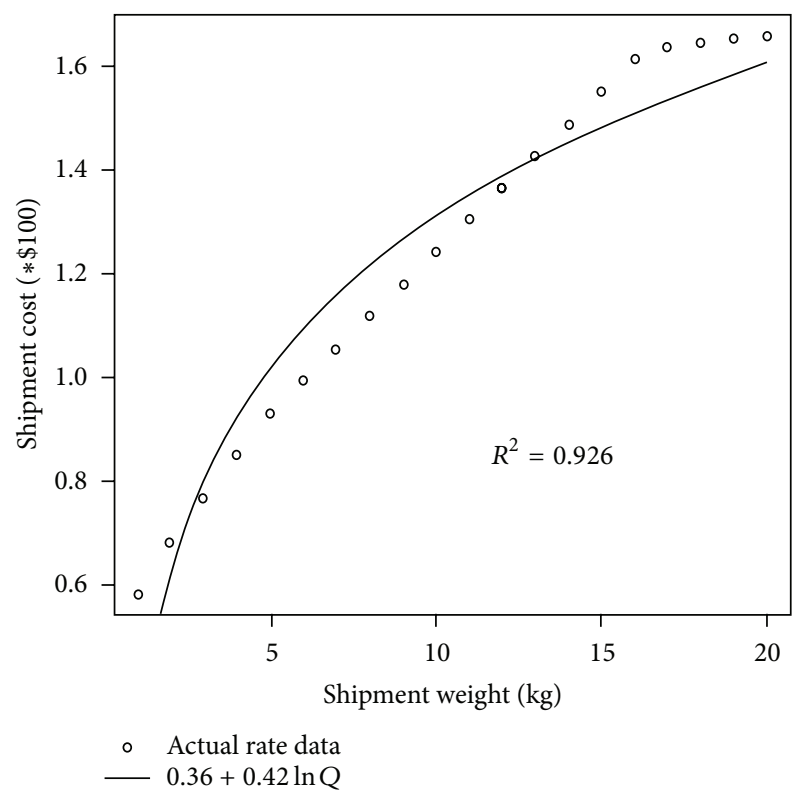

FIgURE 1: The fitted regression model for the data set of UPS worldwide expedited service at zone 7 .

encountering fierce and diverse market circumstances. First, we construct the vendor's expected profit $E(Q)$ :

$$
\begin{aligned}
E(Q)= & p E(\min \{Q, D\})+s E(Q-D)^{+}-c Q \\
& -\{a+b \ln [E(\min \{Q, D\})]\} \\
& -\left\{a+b \ln \left[E(Q-D)^{+}\right]\right\} \\
= & p E(\min \{Q, D\})+s E(Q-D)^{+}-c Q-2 a \\
& -b \ln \left\{E(\min \{Q, D\}) E(Q-D)^{+}\right\} .
\end{aligned}
$$

Then, according to the relationships of $\min \{Q, D\}=D-$ $(D-Q)^{+}$and $(Q-D)^{+}=(Q-D)+(D-Q)^{+}$, we further rewrite (1):

$$
\begin{aligned}
E(Q)= & (p-s) \mu-(p-s) E(D-Q)^{+} \\
& -(c-s) Q-2 a \\
& -b \ln \left\{\left[\mu-E(D-Q)^{+}\right]\left[Q-\mu+E(D-Q)^{+}\right]\right\} .
\end{aligned}
$$

For developing an optimal order quantity for the vendor to sustain its lower bound of maximized expected profit $E(Q)$, we consider $G$, the distribution of $D$, to be under the worst possible distribution $\vartheta$. Therefore, based on Gallego and
Moon's Lemma 1 in [7], we have the lower bound of expected profit $E(Q)$ for the vendor:

$$
\begin{aligned}
E(Q) \geq & (p-s) \mu-(p-s) \\
& \times \frac{\left[\sigma^{2}+(Q-\mu)^{2}\right]^{1 / 2}-(Q-\mu)}{2} \\
& -(c-s) Q-2 a+2 b \ln 2 \\
& -b \ln \left\{-\mu^{2}-1+Q^{2}+2 \mu\left[\sigma^{2}+(Q-\mu)^{2}\right]^{1 / 2}\right\} .
\end{aligned}
$$

Lemma 1 (see [7]). Under the worst possible distribution 9 , the upper bound of expected value for the positive part of $Q-D$ is

$$
E(D-Q)^{+} \leq \frac{\left[\sigma^{2}+(Q-\mu)^{2}\right]^{1 / 2}-(Q-\mu)}{2} .
$$

Let the right-hand side term of (3) be a continuous function with respect to $Q$; then, first and second derivatives of $E(Q)$ are elaborately derived as follows:

$$
\begin{aligned}
\frac{d E(Q)}{d Q}= & \frac{p+s-2 c}{2}-\frac{(p-s)(Q-\mu)}{2\left[\sigma^{2}+(Q-\mu)^{2}\right]^{1 / 2}} \\
& -b \frac{2 Q+2 \mu(Q-\mu)\left[\sigma^{2}+(Q-\mu)^{2}\right]^{-1 / 2}}{-1-\mu^{2}+Q^{2}+4 \mu\left[\sigma^{2}+(Q-\mu)^{2}\right]^{1 / 2}}, \\
\frac{d^{2} E(Q)}{d Q^{2}}= & -\frac{(p-s) \sigma^{2}}{2\left[\sigma^{2}+(Q-\mu)^{2}\right]^{3 / 2}} \\
& -b\left\{\begin{array}{l}
-2+2 \mu^{2}-2 Q^{2} \\
+ \\
+4 \mu\left[\sigma^{2}+(Q-\mu)^{2}\right]^{1 / 2} \\
+\frac{2 \mu\left(-1-\mu^{2}+4 \mu Q-3 Q^{2}\right)}{\left[\sigma^{2}+(Q-\mu)^{2}\right]^{1 / 2}} \\
-\frac{8 \mu^{2}(Q-\mu)^{2}}{\sigma^{2}+(Q-\mu)^{2}} \\
\left.+\frac{(Q-\mu)^{2}\left(2 \mu^{3}+2 \mu-2 \mu Q^{2}\right)}{\left[\sigma^{2}+(Q-\mu)^{2}\right]^{3 / 2}}\right\} \\
-1-\mu^{2}+Q^{2}+4 \mu\left[\sigma^{2}+(Q-\mu)^{2}\right]^{1 / 2}
\end{array}\right\}
\end{aligned} .
$$

Obviously, $d^{2} E(Q) / d Q^{2}$ in (6) is not necessarily being negative. It implies that the generally explicit and analytical close form for the optimal order quantity $\max \left\{Q^{*}, Q^{s}\right\}$ with the least of maximized expected profits is not available. Therefore, there is a need to develop an efficient search procedure to obtain the optimal order quantity $Q^{*}$ and its corresponding lower bound of maximized expected profit $E\left(Q^{*}\right)$. 
TABLE 1: The optimal order quantity using Newton's optimization approach.

\begin{tabular}{lccccc}
\hline Iteration $i$ & $Q_{i}$ & $f^{\prime}\left(Q_{i}\right)$ & $f^{\prime \prime}\left(Q_{i}\right)$ & $f^{\prime}\left(Q_{i}\right) / f^{\prime \prime}\left(Q_{i}\right)$ & 0.281 \\
\hline 0 & 9 & -0.695 & -2.471 & -0.017 & 8.719 \\
1 & 8.719 & 0.030 & -1.729 & 0.008 & 8.736 \\
2 & 8.736 & -0.014 & -1.804 & -0.003 & 8.728 \\
3 & 8.728 & 0.005 & -1.772 & $\mathbf{0 . 0 0 0}$ & 8.731 \\
$\mathbf{4}$ & $\mathbf{8 . 7 3 1}$ & $-\mathbf{0 . 0 0 0}$ & $-\mathbf{1 . 7 8 5}$ & $\mathbf{8 . 7 3 1}$ \\
\hline
\end{tabular}

\section{An Efficient Solution Procedure for $Q^{*}$ and $E\left(Q^{*}\right)$}

Step 1. Start from $i=0$, let initial order quantity $Q_{0}=\mu$, and set the allowable tolerance $\varepsilon$, for example, the acceptable "precision" or "accuracy" selected by the decision maker for the optimal decision policy.

Step 2. Perform Newton's approach (see Hillier and Lieberman [24, pp. 555-557]) to seeking the optimal order quantity of $Q$.

$$
\begin{aligned}
& \text { Let } Q_{i+1}=Q_{i}-\left(f^{\prime}\left(Q_{i}\right) / f^{\prime \prime}\left(Q_{i}\right)\right) \text {. According to (5), we set } \\
& \begin{aligned}
f^{\prime}\left(Q_{i}\right)= & \frac{p+s-2 c}{2}-\frac{(p-s)\left(Q_{i}-\mu\right)}{2\left[\sigma^{2}+\left(Q_{i}-\mu\right)^{2}\right]^{1 / 2}} \\
& -b \frac{2 Q_{i}+2 \mu\left(Q_{i}-\mu\right)\left[\sigma^{2}+\left(Q_{i}-\mu\right)^{2}\right]^{-1 / 2}}{Q_{i}^{2}-\mu^{2}-1+2 \mu\left[\sigma^{2}+\left(Q_{i}-\mu\right)^{2}\right]^{1 / 2}}
\end{aligned}
\end{aligned}
$$

From (6), we set

$$
\begin{aligned}
f^{\prime \prime}\left(Q_{i}\right)=- & \frac{(p-s) \sigma^{2}}{2\left[\sigma^{2}+\left(Q_{i}-\mu\right)^{2}\right]^{3 / 2}} \\
-b\{- & 2 Q_{i}^{2}-2+2 \mu^{2}+4 \mu\left[\sigma^{2}+\left(Q_{i}-\mu\right)^{2}\right]^{1 / 2} \\
& +\frac{2 \mu\left(4 \mu Q_{i}-3 Q_{i}^{2}-\mu^{2}-1\right)}{\left[\sigma^{2}+\left(Q_{i}-\mu\right)^{2}\right]^{1 / 2}} \\
& +\frac{\left(Q_{i}-\mu\right)^{2}\left(2 \mu^{3}+2 \mu-2 \mu Q_{i}^{3}\right)}{\left[\sigma^{2}+\left(Q_{i}-\mu\right)^{2}\right]^{3 / 2}} \\
& \left.-\frac{8 \mu^{2}\left(Q_{i}-\mu\right)^{2}}{\sigma^{2}+\left(Q_{i}-\mu\right)^{2}}\right\} .
\end{aligned}
$$

Stop the search when $\left|Q_{i+1}-Q_{i}\right| \leq \varepsilon$, so the optimal order quantity $Q^{*}$ can be found at the value $Q_{i+1}$.

Step 3. For verifying adequacy of Newton's method, substitute $Q^{*}$ into (6); if $d^{2} E\left(Q^{*}\right) / d Q^{* 2}<0$ meaning Newton's method is satisfactory, then the final solution is $Q^{*}$ whose $E\left(Q^{*}\right)$ is the vendor's lower bound of maximized expected profit; otherwise, go to Step 4 to perform the bisection optimization method.

Step 4. Select $l$, a quantifiable order quantity. Start $i=0$ and let $\left[Q_{0}^{s}, Q_{0}^{*}\right]$ be the initial searching interval, where $Q_{0}^{s}=\exp (1-$ $a / b)$ is the regulated minimal quantity level of delivery for the transportation cost tc $(Q)=a+b \ln Q$ and $Q_{0}^{*}=Q^{*}$.

Step 5. If $\left|Q_{i}^{*}-Q_{i}^{s}\right|<l$, then stop; the optimal order quantity is $Q_{i}^{* *}=\left(Q_{i}^{s}+Q_{i}^{*}\right) / 2$ along with the lower bound of maximal expected profit $E\left(Q_{i}^{* *}\right)$; otherwise, let $Q_{i}^{b}=\left(Q_{i}^{s}+Q_{i}^{*}\right) / 2$.

Step 6. If $E\left(Q_{i}^{b}\right) \geq E\left(Q_{i}^{*}\right)$, then $Q_{i+1}^{*}=Q_{i}^{b}$ and $Q_{i+1}^{s}=Q_{i}^{*}$; otherwise, $Q_{i+1}^{*}=Q_{i}^{*}$ and $Q_{i+1}^{s}=Q_{i}^{b}$. Go back Step 5 with $i=i+1$.

To demonstrate the efficient solution procedure for the DFNP incorporating the explicit transportation cost, a numerical example is illustrated.

3.1. Finding $Q^{*}$ and $E\left(Q^{*}\right)$. A chosen product has demand mean $\mu=9 \mathrm{~kg}$ and standard deviation $\sigma=0.5$. Its unit cost is $c=\$ 3.5 / \mathrm{kg}$, unit selling price $p=\$ 5 / \mathrm{kg}$, and unit salvage value $s=\$ 2.5 / \mathrm{kg}$. Including fuel and handling charges, on-site data of the transportation cost collected from UPS worldwide expedited service at zone 7, from Europe to Taiwan, are $0.58,0.69,0.77,0.85,0.93,1.00,1.06,1.12,1.18$, $1.24,1.31,1.37,1.43,1.49,1.55,1.61,1.64,1.65,1.66$, and 1.66 for shipment weight of $1,2, \ldots, 20 \mathrm{~kg}$, respectively. For clarity of description, the costs considered here are all rounded down to a 45-hundred US dollar-scale. By fitting the data through the nonlinear regression model, we have an empirical tampering function $\operatorname{tc}(Q)=0.36+0.42 \ln Q$ shown in Figure 1 with $R^{2}=0.926$. We conclude that the fitted function provides high fidelity to represent the actual data.

Then, we follow the proposed search procedure.

Step 1. From $n=0$ and $i=0$, set $Q_{0}=\mu=9$ and $\varepsilon=10^{-3}$.

Step 2. When $n=1$, we have $Q_{1}=Q_{0}-\left(f^{\prime}\left(Q_{0}\right) / f^{\prime \prime}\left(Q_{0}\right)\right)=$ 9.211. In this case, $\left|Q_{1}-Q_{0}\right|>0.001$, so continue Newton's search until reaching $\left|Q_{i+1}-Q_{i}\right| \leq 0.001$. Then the optimal order quantity $Q^{*}=Q_{i+1}$. The searching details are listed in Table 1 .

Step 3. The optimal order quantity $Q^{*}=8.731$ (the condition $d^{2} E\left(Q^{*}\right) / d Q^{* 2}=-1.783<0$ holds true). Substituting $Q^{*}=8.731$ and known parameters into (5), we obtain lower bound of maximized expected profit $E\left(Q^{*}\right)$, which is $\$ 11.899$. 
TABLE 2: The computational results with fixed values of $p=5$ and $s=2.5$.

\begin{tabular}{|c|c|c|c|c|c|c|c|}
\hline \multirow{2}{*}{ Policy } & \multicolumn{4}{|c|}{ Parameters setting } & \multirow{2}{*}{$\begin{array}{c}\text { Our proposed policy } \\
E\left(Q^{*}\right)\end{array}$} & \multirow{2}{*}{$\begin{array}{c}\text { Gallego and Moon [7] } \\
E\left(Q^{*}\right)\end{array}$} & \multirow{2}{*}{$\begin{array}{c}\text { Profit gain } \\
(\%) \\
\end{array}$} \\
\hline & $\mu$ & $\sigma$ & $c$ & $\mathrm{tc}(Q)$ & & & \\
\hline 1 & 7 & 0.4 & 3 & $0.36+0.42 \ln Q$ & $12.659(6.824)$ & $12.454(7.300)$ & 1.62 \\
\hline 2 & 11 & 0.4 & 3 & $0.36+0.42 \ln Q$ & $20.470(10.863)$ & $20.262(11.300)$ & 1.02 \\
\hline 3 & 7 & 0.6 & 3 & $0.36+0.42 \ln Q$ & $12.236(6.98)$ & $12.086(7.450)$ & 1.22 \\
\hline 4 & 11 & 0.6 & 3 & $0.36+0.42 \ln Q$ & $20.040(10.700)$ & $19.893(11.450)$ & 0.73 \\
\hline 5 & 7 & 0.4 & 4 & $0.36+0.42 \ln Q$ & $5.939(6.503)$ & $5.533(7.082)$ & 6.84 \\
\hline 6 & 11 & 0.4 & 4 & $0.36+0.42 \ln Q$ & $9.736(10.516)$ & $9.339(11.082)$ & 4.08 \\
\hline 7 & 7 & 0.6 & 4 & $0.36+0.42 \ln Q$ & $5.476(6.509)$ & $5.102(7.122)$ & 6.82 \\
\hline 8 & 11 & 0.6 & 4 & $0.36+0.42 \ln Q$ & $9.271(10.509)$ & $8.907(11.122)$ & 3.93 \\
\hline 9 & 7 & 0.4 & 3 & $0.31+0.56 \ln Q$ & $12.764(6.705)$ & $12.411(7.300)$ & 2.76 \\
\hline 10 & 11 & 0.4 & 3 & $0.31+0.56 \ln Q$ & $20.511(10.754)$ & $20.155(11.300)$ & 1.73 \\
\hline 11 & 7 & 0.6 & 3 & $0.31+0.56 \ln Q$ & $12.250(6.858)$ & $11.988(7.450)$ & 2.13 \\
\hline 12 & 11 & 0.6 & 3 & $0.31+0.56 \ln Q$ & $19.987(10.881)$ & $19.731(11.450)$ & 1.28 \\
\hline 13 & 7 & 0.4 & 4 & $0.31+0.56 \ln Q$ & $6.160(6.444)$ & $5.561(7.082)$ & 9.74 \\
\hline 14 & 11 & 0.4 & 4 & $0.31+0.56 \ln Q$ & $9.889(10.435)$ & $9.303(11.082)$ & 5.93 \\
\hline 15 & 7 & 0.6 & 4 & $0.31+0.56 \ln Q$ & $5.605(6.428)$ & $5.075(7.122)$ & 9.46 \\
\hline \multirow[t]{2}{*}{16} & 11 & 0.6 & 4 & $0.31+0.56 \ln Q$ & $9.331(10.414)$ & 8.814(11.122) & 5.54 \\
\hline & & & & & & Average & 4.05 \\
\hline
\end{tabular}

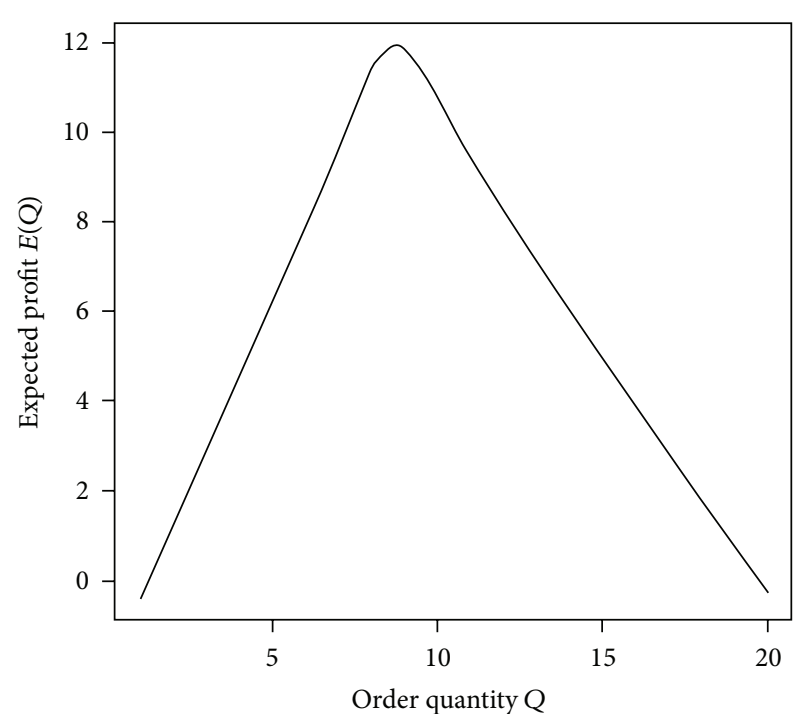

FIGURE 2: Illustration of the expected profit with respect to order quantity $Q$.

Figure 2 concavely exhibits $E\left(Q^{*}\right)$ with respect to a wide range of $Q^{*}$.

3.2. Models Comparison. For models comparison, we implement the DFNP based on Gallego and Moon [7] whose model does not reckon the transportation cost and perform the similar searching procedure described in Section 3. Their model obtains the optimal order quantity $Q^{*}=8.731$ with the lower bound of maximized expected profit $E\left(Q^{*}\right)=\$ 11.752$. In this case, our proposed model in consideration of the transportation cost has manifested $(11.899-11.752) / 11.899=$ $1.2 \%$ of gains in $E\left(Q^{*}\right)$.

\section{Sensitivity Analyses and Comparative Studies}

Furthermore, we apply a $2^{4}$ factorial design to investigate sensitivity of parameters. They are set as follows. Let the unit selling price be $p=\$ 5 / \mathrm{kg}$ and the unit salvage value be $s=\$ 2.5 / \mathrm{kg}$; two levels are selected for each of the four parameters; that is, mean $\mu \in[7,11]$, standard deviation $\sigma \in$ $[0.1,1]$, unit product cost $c \in[3,4]$, and the transportation cost $\mathrm{tc}(Q) \in[0.36+0.42 \ln Q, 0.31+0.56 \ln Q]$ whose selected levels are based on fitting another data set gathered from UPS's transportation cost (worldwide express saver at zone 7, from Europe to Taiwan), US\$ 0.66, 0.78, 0.88, 0.98, $1.08,1.15,1.23,1.31,1.39,1.47,1.55,1.62,1.70,1.79,1.87,1.94$, $2.01,2.09,2.17$, and 2.25 , respectively, for shipment weight of $1,2,3, \ldots, 20 \mathrm{~kg}$.

Table 2 lists $E\left(Q^{*}\right)$ along with $Q^{*}$ for our proposed model in the 6th column and Gallego and Moon's model [7] in the 7th column. First, this sensitivity analysis demonstrates significant correlations among the parameters whose simultaneous consideration is imperative for the proposed optimal policy. Moreover, in contrast to Gallego and Moon's model, the percentages of the profit gain obtained from our proposed model are listed in the 8th column. Apparently, our proposed model outperforms Gallego and Moon's model in every policy; especially, in the ordering policies 13 and 15, the profit advance can be more than $9.4 \%$; on average, our proposed policy provides the return gain as much as $4 \%$ as opposed to that of the Gallego and Moon's model.

In views of the impact of transportation cost on the DFNP as well as the gains elicited from our proposed policies, we 
then extend contemplation of the transportation cost into several practical inventory cases such as fixed ordering cost, random yield, and a multiproduct case.

\section{The Fixed Ordering Cost Case with Transportation Cost}

Let a vendor have an initial inventory $I(I \geq 0)$ prior to placing an order $Q>0$, where ordering cost $A$ is fixed for any size of order. Let $r$ denote the reorder point, known as an inventory level when the order is submitted. Let $S=I+Q$ be end inventory level, an inventory level after receiving the order.

Similarly, $\min \{S, D\}$ units are sold; $S-D$ units are salvaged. For an $(r, S)$ inventory replenishment policy in consideration of the transportation cost, expected profit $E(S)$ is constructed as

$$
\begin{aligned}
E(S)= & p E(\min \{S, D\})+s E(S-D)^{+} \\
& -c(S-I)-A 1_{[S>I]}-\{a+b \ln [E(\min \{S, D\})]\} \\
& -\left\{a+b \ln \left[E(S-D)^{+}\right]\right\} \\
E(S)= & (p-s) \mu-(p-s) E(D-S)^{+} \\
& -(c-s) S+c I-A I_{[S>I]}-2 a \\
& -b \ln \left\{\left[\mu-E(D-S)^{+}\right]\left[S-\mu+E(D-S)^{+}\right]\right\},
\end{aligned}
$$

where $I_{[S>I]}= \begin{cases}1 & \text { if } S>I \text {, } \\ 0 & \text { otherwise. }\end{cases}$

According to Lemma 1, the expression can be simplified as $\min _{S \geq I}\left\{A I_{[S>I]}+J(S)\right\}$, where

$$
\begin{aligned}
J(S)= & -(p-s) \mu+(p-s) \frac{\left[\sigma^{2}+(S-\mu)^{2}\right]^{1 / 2}-(S-\mu)}{2} \\
& +(c-s) S-c I+2 a-2 b \ln 2 \\
& +b \ln \left\{-\mu^{2}-1+S^{2}+2 \mu\left[\sigma^{2}+(S-\mu)^{2}\right]^{1 / 2}\right\} .
\end{aligned}
$$

The relationship of $S=I+Q$ implies that acquiring the optimal end inventory level of $S$ for the fixed ordering cost model is equivalent to having optimal order quantity of $Q$ for the single-product model. Clearly, because $I\langle S, J(I)\rangle A+$ $J(S)$. For determining the optimal reorder point of $r, J(r)=$ $A+J(S)$ is set. Then, we have

$$
\begin{aligned}
& \frac{p-s}{2}\left\{\left[\sigma^{2}+(r-\mu)^{2}\right]^{1 / 2}-r\right\}+(c-s) r \\
& +b \ln \left\{-\mu^{2}-1+r^{2}+2 \mu\left[\sigma^{2}+(r-\mu)^{2}\right]^{1 / 2}\right\} \\
& -A-\frac{p-s}{2}\left\{\left[\sigma^{2}+(S-\mu)^{2}\right]^{1 / 2}-S\right\}-(c-s) S \\
& -b \ln \left\{-\mu^{2}-1+S^{2}+2 \mu\left[\sigma^{2}+(S-\mu)^{2}\right]^{1 / 2}\right\}=0 .
\end{aligned}
$$

Furthermore, we develop a solution procedure to determine the optimal reorder point.

Step 1. By performing the solution procedure for the optimal order quantity in Section 3, we first obtain $Q^{*}$. Then let $Q^{*}$ be the end inventory level $S$, where $I$ is set to be 0 for brevity.

Step 2. Start $i=0$, set the initial reorder point $r_{0}$ to be $S$, and determine the allowable tolerance $\varepsilon$ for accuracy of the final result.

Step 3. Perform Newton's search (see Grossman [25, pp.228]) to compute the optimal reorder level of $r$. That is, $r_{i+1}=r_{i}$ $\left(f\left(r_{i}\right) / f^{\prime}\left(r_{i}\right)\right)$, where

$$
\begin{aligned}
f\left(r_{i}\right)= & \frac{p-s}{2}\left\{\left[\sigma^{2}+\left(r_{i}-\mu\right)^{2}\right]^{1 / 2}-r_{i}\right\}+(c-s) r_{i} \\
& +b \ln \left\{-\mu^{2}-1+r_{i}^{2}+2 \mu\left[\sigma^{2}+\left(r_{i}-\mu\right)^{2}\right]^{1 / 2}\right\} \\
& -A-\frac{p-s}{2}\left\{\left[\sigma^{2}+(S-\mu)^{2}\right]^{1 / 2}-S\right\}-(c-s) S \\
& -b \ln \left\{-\mu^{2}-1+S^{2}+2 \mu\left[\sigma^{2}+(S-\mu)^{2}\right]^{1 / 2}\right\} \\
f^{\prime}\left(r_{i}\right)= & \frac{2 c-p-s}{2}+\frac{(p-s)\left(r_{i}-\mu\right)}{2\left[\sigma^{2}+\left(r_{i}-\mu\right)^{2}\right]^{1 / 2}} \\
& +b \frac{2 r_{i}+2 \mu\left(r_{i}-\mu\right)\left[\sigma^{2}+\left(r_{i}-\mu\right)^{2}\right]^{-1 / 2}}{-\mu^{2}-1+r_{i}^{2}+2 \mu\left[\sigma^{2}+\left(r_{i}-\mu\right)^{2}\right]^{1 / 2}} .
\end{aligned}
$$

Stop the search when $\left|r_{i+1}-r_{i}\right| \leq \varepsilon$. Then, the optimal order quantity is $r_{i+1}$.

Step 4. The optimal policy is to order up to $S$ units if the initial inventory is less than $r$ and not to order otherwise.

5.1. An Example. Continuing the numerical example in Section 3, we assume that the ordering cost is given by $A=$ $\$ 0.3$. Using the above solution procedure, we find that the optimal reorder level of $r$ is 8.210 and the end inventory level $S=8.731$.

\section{The Random Yield Case with Transportation Cost}

Suppose random variable $G(Q)$ expresses the number of good units produced from ordered quantity $Q$, where each good unit being ordered or produced has an equal probability of $\rho$. Thus, $G(Q)$ is a binomial random variable with mean $Q \rho$ and variance $Q \rho q$, where $q=1-\rho$. Let $m$ be the price markup rate and $d$ the discount rate, so unit selling price $p=(1+m) c / \rho$, 
and salvage value $s=(1-d) c / \rho$. Thus, the expected profit in (1) can be rewritten as

$$
\begin{aligned}
E(Q)= & p E(\min \{G(Q), D\})+s E(G(Q)-D)^{+}-c Q-2 a \\
& -b \ln \left\{E(\min \{G(Q), D\}) E(G(Q)-D)^{+}\right\} \\
= & \frac{c}{\rho}\left\{(m+d) \mu-(m+d) E[D-G(Q)]^{+}\right. \\
& -(\rho+d-1) Q\}-2 a \\
- & b \ln \left\{\left[\mu-E[D-G(Q)]^{+}\right]\right. \\
& \left.\times\left[Q-\mu+E[D-G(Q)]^{+}\right]\right\} .
\end{aligned}
$$

Applying Lemma 1 to this case, we have

$$
E[D-G(Q)]^{+} \leq \frac{\left[\sigma^{2}+\rho q Q+(\rho Q-\mu)^{2}\right]^{1 / 2}-(\rho Q-\mu)}{2} .
$$

Substituting the above relationship into (13), we have lower bound of the expected profit in this case. Consider

$$
\begin{aligned}
E(Q) \geq \frac{c}{\rho}\{( & (m+d) \mu-(m+d) \\
& \times \frac{\left[\sigma^{2}+\rho q Q+(\rho Q-\mu)^{2}\right]^{1 / 2}-(\rho Q-\mu)}{2} \\
- & (\rho+d-1) Q\}-2 a+2 b \ln 2 \\
-b \ln \left\{2(\rho Q+\mu)(1-\rho) Q-\sigma^{2}-\rho q Q\right. & +2(\mu+\rho Q-Q) \\
& \left.\times\left[\sigma^{2}+\rho q Q+(\rho Q-\mu)^{2}\right]^{1 / 2}\right\} .
\end{aligned}
$$

The right-hand side of (15) is a continuous function in terms of $Q$. Then, first and second derivatives of $E(Q)$ can be derived as

$$
\begin{aligned}
& \frac{d E(Q)}{d Q} \\
& =-\frac{c(m+d)}{2}\left[\frac{1}{2} X^{-1 / 2}(q-2 \mu+2 \rho Q)-1\right]-\frac{c}{\rho}(\rho+d-1) \\
& \quad-b\left(2(1-\rho)(\mu+2 \rho Q)-\rho q-2(1-\rho) X^{1 / 2}\right. \\
& \left.\quad+\rho(\mu+\rho Q-Q)(q-2 \mu+2 \rho Q) X^{-1 / 2}\right) \\
& \quad \times\left(2(\rho Q+\mu)(1-\rho) Q-\sigma^{2}\right. \\
& \left.\quad-\rho q Q+2(\mu+\rho Q-Q) X^{1 / 2}\right)^{-1}
\end{aligned}
$$

where $X=\sigma^{2}+\rho q Q+(\rho Q-\mu)^{2}$

$$
\begin{aligned}
\frac{d^{2} E(Q)}{d Q^{2}}= & -\frac{c(m+d)}{2}\left[\frac{-\rho}{4}(q-2 \mu+2 \rho Q)^{2} X^{-3 / 2}+\rho X^{-1 / 2}\right] \\
& -b \frac{Y^{\prime} Z-Y Z^{\prime}}{Z^{2}},
\end{aligned}
$$

where

$$
\begin{aligned}
Y=2 & (1-\rho)(\mu+2 \rho Q)-\rho q-2(1-\rho) X^{1 / 2} \\
& +\rho(\mu+\rho Q-Q)(q-2 \mu+2 \rho Q) X^{-1 / 2}, \\
Z=2 & (\rho Q+\mu)(1-\rho) Q-\sigma^{2}-\rho q Q \\
& +2(\mu+\rho Q-Q) X^{1 / 2}, \\
Y^{\prime}= & 4 \rho(1-\rho)-2 \rho \\
& \times[(1-\rho)(q-2 \mu+2 \rho Q)-\rho(\mu+\rho Q-Q)] X^{-1 / 2} \\
& -\frac{\rho^{2}}{2}(\mu+\rho Q-Q)(q-2 \mu+2 \rho Q)^{2} X^{-3 / 2}, \\
Z^{\prime}= & 2(1-\rho)(2 \rho Q+\mu)-\rho q \\
& -2(1-\rho) X^{1 / 2}+\frac{\rho}{4}(\mu+\rho Q-Q) \\
& \times(q-2 \mu+2 Q) X^{-1 / 2} .
\end{aligned}
$$

Obviously, $d^{2} E(Q) / d Q^{2}$ is not necessarily being negative.

Similarly, we develop a solution procedure to find the optimal order quantity in this random yield case.

Step 1. Start $i=0$ and $Q_{0}=\mu$. Set the allowable tolerance $\varepsilon$.

Step 2. Perform Newton's search (see Hillier and Lieberman [24], pp.555-557) to compute the optimal order quantity $Q$. That is, $Q_{i+1}=Q_{i}-\left(f^{\prime}\left(Q_{i}\right) / f^{\prime \prime}\left(Q_{i}\right)\right)$, where $f^{\prime}\left(Q_{i}\right)$ and $f^{\prime \prime}\left(Q_{i}\right)$ stand for (16) and (17), respectively. Stop the search when $\left|Q_{i+1}-Q_{i}\right| \leq \varepsilon$. The optimal order quantity is $Q_{i+1}$.

Step 3. For verifying adequacy of Newton's method, substitute $Q_{i+1}$ into (19); if $d^{2} E\left(Q_{i+1}\right) / d Q_{i+1}^{2}<0$, representing Newton's method, is satisfactory, then the final solution is $Q^{*}=Q_{i+1}$ whose $E\left(Q^{*}\right)$ is the vendor's lower bound of the maximized expected profit; otherwise, go to Step 4 to perform the bisection optimization method.

Step 4. Select $l$, a quantifiable order quantity. Start $i=0$ and let $\left[Q_{0}^{s}, Q_{0}^{*}\right]$ be the initial searching interval, where $Q_{0}^{s}=\exp (1-$ $a / b)$ is the regulated minimal quantity level of delivery for the transportation cost tc $(Q)=a+b \ln Q$ and $Q_{0}^{*}=Q^{*}$.

Step 5. If $\left|Q_{i}^{*}-Q_{i}^{s}\right|<l$, then stop; the optimal order quantity is $Q_{i}^{* *}=\left(Q_{i}^{s}+Q_{i}^{*}\right) / 2$ along with $E\left(Q_{i}^{* *}\right)$, the lower bound of maximal expected profit; otherwise, let $Q_{i}^{b}=\left(Q_{i}^{s}+Q_{i}^{*}\right) / 2$.

Step 6. If $E\left(Q_{i}^{b}\right) \geq E\left(Q_{i}^{*}\right)$, then $Q_{i+1}^{*}=Q_{i}^{b}$ and $Q_{i+1}^{s}=Q_{i}^{*}$; otherwise, $Q_{i+1}^{*}=Q_{i}^{*}$ and $Q_{i+1}^{s}=Q_{i}^{b}$. Go back Step 5 with $i=i+1$. 
6.1. An Example. We continue Section 3. We assume that, for each unit of $Q$, the probability of being good is $\rho=0.9$. We find the optimal order quantity $Q^{*}=10.403$, and the lower bound of the maximum expected profit $E\left(Q^{*}\right)$ is 14.573 . The condition $d^{2} E\left(Q_{i+1}\right) / d Q_{i+1}^{2}=-0.916<0$ is satisfactory. In contrast, the order quantity placed on the product with perfect quality can be computed as much as 8.731 , which is smaller than $Q^{*}=10.403$. Apparently, in therandom yield case the order quantity is increased to provide safeguard against a possible shortage.

\section{The Multiproduct Case with Transportation Cost}

We now study a multiproduct newsboy problem in the presence of a budget constraint, also known as the stochastic product-mixed problem [26]. Suppose that each product $j$, for $j=1, \ldots, N$, has order quantity $Q_{j}$ received from either purchasing or manufacturing, where a limited budget is allocated due to the limited production capacity in the system. That is, the total purchasing or manufacturing cost for all the $N$ competing products cannot exceed allotted budget $B$. Denote that each item's unit cost of the $j$ th product is $c_{j}$, its unit selling price is $p_{j}$, and its unit salvage value is $s_{j}$. For the $j$ th product's demand, its mean and variance are denoted by $\mu_{j}$ and $\sigma_{j}^{2}$, respectively.

In the sequel, under the distribution-free demand jointed with the explicit transportation cost, the vendor is in need of deciding the optimal order quantities for $N$ competing products whose total purchasing or manufacturing cost does not exceed the allocated budge $B$, where he/she guarantees to possess the least of all possible maximum expected profits.

For solving this problem, we first extend the single product case in (3) to have lower bound of expected profit $E\left(Q_{1}, \ldots, Q_{N}\right)$ for the vendor, provided that the individual order quantity of $Q_{1}, Q_{2}, \ldots$, and $Q_{N}$ is affected by the budget constraint $B$. For the vendor to secure the least amount of the maximum expected profit over various situations of market, we maximize (19) with a budget constraint expressed in (20) to determine the optimal order quantities $Q_{1}^{*}, Q_{2}^{*}, \ldots$, and $Q_{N}^{*}$ :

$$
\begin{gathered}
\max _{Q_{1}, \ldots, Q_{N}} \sum_{j=1}^{N}\left\{\left(p_{j}-s_{j}\right) \frac{\left(Q_{j}+\mu_{j}\right)-\left[\sigma_{j}^{2}+\left(Q_{j}-\mu_{j}\right)^{2}\right]^{1 / 2}}{2}\right. \\
-\left(c_{j}-s_{j}\right) Q_{j}-2 a+2 b \ln 2 \\
-b \ln \left\{-\mu_{j}^{2}-1+Q_{j}^{2}\right. \\
\left.\left.+2 \mu\left[\sigma_{j}^{2}+\left(Q_{j}-\mu_{j}\right)^{2}\right]^{1 / 2}\right\}\right\},
\end{gathered}
$$

Subject to $\sum_{j=1}^{N} c_{j} Q_{j} \leq B$.
We further transfer the problem into an unconstrained optimization equation:

$$
\begin{aligned}
L\left(Q_{1}, \ldots, Q_{N}, \lambda\right) & \\
=\sum_{j=1}^{N}\{ & \left\{\left(p_{j}-s_{j}\right) \frac{\left(Q_{j}+\mu_{j}\right)-\left[\sigma_{j}^{2}+\left(Q_{j}-\mu_{j}\right)^{2}\right]^{1 / 2}}{2}\right. \\
& -\left(c_{j}-s_{j}\right) Q_{j}-2 a+2 b \ln 2 \\
& -b \ln \left\{-\mu_{j}^{2}-1+Q_{j}^{2}\right. \\
+\lambda\left(\sum_{j=1}^{N} c_{j} Q_{j}-B\right), & \left.\left.+2 \mu_{j}\left[\sigma_{j}^{2}+\left(Q_{j}-\mu_{j}\right)^{2}\right]^{1 / 2}\right\}\right\}
\end{aligned}
$$

where $\lambda$ is the Lagrange multiplier. Hence, we have

$$
\begin{aligned}
& \frac{\partial L\left(Q_{1}, \ldots, Q_{N}, \lambda\right)}{\partial Q_{j}} \\
& =\frac{p_{j}+s_{j}-2 c_{j}}{2}-\frac{\left(p_{j}-s_{j}\right)\left(Q_{j}-\mu_{j}\right)}{2\left[\sigma_{j}^{2}+\left(Q_{j}-\mu_{j}\right)^{2}\right]^{1 / 2}} \\
& -b \frac{2 Q_{j}+2 \mu_{j}\left(Q_{j}-\mu_{j}\right)\left[\sigma_{j}^{2}+\left(Q_{j}-\mu_{j}\right)^{2}\right]^{-1 / 2}}{-1-\mu^{2}+Q_{j}^{2}+4 \mu_{j}\left[\sigma_{j}^{2}+\left(Q_{j}-\mu_{j}\right)^{2}\right]^{1 / 2}} .
\end{aligned}
$$

To find the optimal order quantities $Q_{1}^{*}, Q_{2}^{*}, \ldots$, and $Q_{N}^{*}$ with maximum $L$, we set $\partial L / \partial Q_{j}=0$. In this case, a line search procedure is developed.

Step 1. For multiple products $N$, let $j=\{1, \ldots, N\}$.

Step 2. Let $\lambda=0$ and perform the solution procedure proposed in Section 3 to find $Q_{j}^{*}$. If $(20)$ is satisfied, go to Step 6; otherwise, go to Step 3.

Step 3. Substituting each of $Q_{1}^{*}, Q_{2}^{*}, \ldots$, and $Q_{N}^{*}$ into (22), their corresponding $\lambda$ can be obtained.

Step 4. Start from the smallest nonnegative $\lambda$, let its corresponding optimal order quantity be 0 (others are intact), and check the condition of (20).

Step 5. If the condition is satisfactory, then we have the final solution $Q_{1}^{*}, Q_{2}^{*}, \ldots$, and $Q_{N}^{*}$; otherwise, select the next smallest nonnegative $\lambda$ to perform the same procedure in Step 4 until (20) is satisfied.

Step 6. Find the least amount of the maximum expected profit $E\left(Q_{1} *, \ldots, Q_{N} *\right)$. 
7.1. An Example. The total budget is $\$ 80$ for the four items. The relevant data are as follows: $c=(3.5,2.5,2.8,0.5), p=(5$, $4,3.2,0.6), s=(2.5,1.2,1.5,0.2), \mu=c(9,8,12,23)$, and $\sigma=$ $c(0.5,1,0.7,1)$. Performing the above procedure, we have the following.

Step 1 . Let $j=\{1,2,3,4\}$.

Step 2. Let $\lambda=0$. We solve the four order quantities by using the solution procedure introduced in Section 3. The optimal order quantities $Q_{1}^{*}=8.731, Q_{2}^{*}=7.762, Q_{3}^{*}=11.072$, and $Q_{4}^{*}$ $=21.243$. Check $\sum_{j=1}^{4} c_{j} Q_{j}^{*}=\$ 92>\$ 80$, where (20) is not satisfied, so we go to Step 3.

Step 3. Performing a simple line search, we increase the optimal value of the Lagrangian multiplier until $\lambda=0.147$. In this case, its corresponding $Q_{3}^{*}$ is set to 0 .

Step 4. Since $\sum_{j=1}^{4} c_{j} Q_{j}^{*}=\$ 61<\$ 80,(20)$ is satisfied.

Step 5. The optimal order quantities are 8.731, 7.762, 0 , and 21.243 and the lower bound of the maximum expected profit is $\$ 21.667$.

\section{Conclusions and Implications}

Models for the distribution-free newsboy problem have been widely introduced over the past two decades to provide the optimal order quantity for securing the vendor with the least amount of the maximum expected profit when facing a variety of situations in modern business environment.

Over the past few years, energy prices have risen significantly so that the transportation of goods has become a vital component for the vendor's logistic-cost function to determine its required purchase quantities. However, impacts of the transportation cost on previous models for the DFNP were inattentive by either overlooking or deeming it as part of implicit components of ordering cost. In this paper, three main contributions along with their managerial implication have been done.

First, we develop the DFNP incorporating the explicit transportation cost into the expected profit function. In particular, the transportation cost is modeled based on the economic theory from transportation disciplines and fitted a nonlinear regression via actual rate data collected from the shipper. In practice, this way has implied that (1) economic trade-off for the optimal transportation cost lies between provided service level and shipped quantity; (2) in the shipment, more weight signifies larger delivery quantity and higher shipment cost; and (3) optimal shipping quantity renders minimum of the transportation cost.

Secondly, since the expected profit function is neither concave nor convex, the optimization problem underlying this generalization is challenging; therefore, we developed analytical and efficient procedures to acquire the optimal policy. As a result of the computational studies, our proposed optimal ordering rules in comparison with the optimal policy recommended by Gallego and Moon [7] increased the lower bound of the maximal expected profit by as much as $4 \%$ on average. This result has demonstrated that the expenditure of the inbound/outbound material transportation has become a critical component of a total annual logistic cost function for determining purchase quantities. Effects of transportation have gained substantial recognition in the DFNP.

Thirdly, according to the results of sensitivity analyses, the parameters, such as demand mean and variance, product's unit cost, and transportation cost, are the key decision variables whose joint reckoning is imperative for the optimal policy proposed. Moreover, we proceed to analyses of several practical inventory cases including fixed ordering cost, random yield, and multiproduct case. These studies further demonstrate the impacts of transportation cost as well as the realized-least profit gains drawn from our recommended policies on the DFNP that explicitly incorporates the transportation cost into consideration. In addition, these numerical findings have implied that joint decision, coordinated operation, or integrated management is crucial in lowering the vendor-and-buyer operating cost as well as balancing a supply-chain operation and structure.

Finally, based on the shipping data sets collected from United Parcel Service (UPS), the transportation cost is modeled using a natural logarithm for a nonlinear regression function in this paper. For future studies, other functional forms may be reckoned to model different transportation costs, such as a step function or a logistic function, to validate a wide variety of applications. Besides, using our proposed model as a basis model in a couple of more advanced studies with certain circumstances, such as the multiproduct newsboy under a value-at-risk and the multiple newsvendors with loss-averse preferences, is intriguing.

\section{Highlights}

(i) We extend previous work on the distribution-free newsboy problem where the vendor's expected profit is in presence of transportation cost.

(ii) The transportation cost is formulated as a function of shipping quantity and modeled as a nonlinear regression form based on UPS's on-site shipping-rate data.

(iii) The comparative studies have demonstrated significant positive impacts by using our proposed methodology whose profit gains in comparison with prior research can be as much as $9 \%$ and $4 \%$ on average.

(iv) The sensitivity analyses jointly reckon the imperative parameters for the optimal policy.

(v) We expand our methodology to several practical inventory cases including fixed ordering cost, random yield, and a multiproduct condition.

\section{Conflict of Interests}

The authors declare that there is no conflict of interests regarding the publication of this paper. 


\section{References}

[1] M. Khouja, "The single-period (news-vendor) problem: literature review and suggestions for future research," Omega, vol. 27, no. 5, pp. 537-553, 1999.

[2] S.-P. Chen and Y.-H. Ho, "Optimal inventory policy for the fuzzy newsboy problem with quantity discounts," Information Sciences, vol. 228, pp. 75-89, 2013.

[3] S. B. Ding, "Uncertain random newsboy problem," Journal of Intelligent and Fuzzy Systems, vol. 26, no. 1, pp. 483-490, 2014.

[4] V. Arshavskiy, V. Okulov, and A. Smirnova, "Newsvendor problem experiments: riskiness of the decisions and learning by experience," International Journal of Business and Social Research, vol. 4, no. 5, pp. 137-150, 2014.

[5] A. Özler, B. Tan, and F. Karaesmen, "Multi-product newsvendor problem with value-at-risk considerations," International Journal of Production Economics, vol. 117, no. 2, pp. 244-255, 2009.

[6] C. X. Wang, “The loss-averse newsvendor game," International Journal of Production Economics, vol. 124, no. 2, pp. 448-452, 2010.

[7] G. Gallego and I. Moon, "Distribution free newsboy problem: review and extensions," Journal of the Operational Research Society, vol. 44, no. 8, pp. 825-834, 1993.

[8] H. K. Alfares and H. H. Elmorra, "The distribution-free newsboy problem: extensions to the shortage penalty case," International Journal of Production Economics, vol. 93-94, pp. 465-477, 2005.

[9] I. Moon and S. Choi, "The distribution free newsboy problem with balking," Journal of the Operational Research Society, vol. 46, no. 4, pp. 537-542, 1995.

[10] X. Cai, S. K. Tyagi, and K. Yang, "Activity-based costing model for MGPD," in Improving Complex Systems Today, pp. 409-416, Springer, London, UK, 2011.

[11] C.-M. Lee and S.-L. Hsu, "The effect of advertising on the distribution-free newsboy problem," International Journal of Production Economics, vol. 129, no. 1, pp. 217-224, 2011.

[12] M. G. Güler, "A note on: 'the effect of optimal advertising on the distribution-free newsboy problem,' International Journal of Production Economics, vol. 148, pp. 90-92, 2014.

[13] J. Kamburowski, "The distribution-free newsboy problem under the worst-case and best-case scenarios," European Journal of Operational Research, vol. 237, no. 1, pp. 106-112, 2014.

[14] J. Mostard, R. de Koster, and R. Teunter, "The distribution-free newsboy problem with resalable returns," International Journal of Production Economics, vol. 97, no. 3, pp. 329-342, 2005.

[15] J. Barry, Rising Transportation Costs-and What to do about Them, Article and White Papers, F. Curtis Barry \& Company, 2013.

[16] S. R. Swenseth and M. R. Godfrey, "Incorporating transportation costs into inventory replenishment decisions," International Journal of Production Economics, vol. 77, no. 2, pp.113-130, 2002.

[17] S. Çetinkaya and C.-Y. Lee, "Optimal outbound dispatch policies: modeling inventory and cargo capacity," Naval Research Logistics, vol. 49, no. 6, pp. 531-556, 2002.

[18] A. Toptal, S. Çetinkaya, and C.-Y. Lee, "The buyer-vendor coordination problem: modeling inbound and outbound cargo capacity and costs," IIE Transactions, vol. 35, no. 11, pp. 9871002, 2003.
[19] A. Toptal and S. Çetinkaya, "Contractual agreements for coordination and vendor-managed delivery under explicit transportation considerations," Naval Research Logistics, vol. 53, no. 5, pp. 397-417, 2006.

[20] J.-L. Zhang, C.-Y. Lee, and J. Chen, "Inventory control problem with freight cost and stochastic demand," Operations Research Letters, vol. 37, no. 6, pp. 443-446, 2009.

[21] A. Toptal, "Replenishment decisions under an all-units discount schedule and stepwise freight costs," European Journal of Operational Research, vol. 198, no. 2, pp. 504-510, 2009.

[22] F. Mutlu and S. Çetinkaya, "An integrated model for stock replenishment and shipment scheduling under common carrier dispatch costs," Transportation Research E: Logistics and Transportation Review, vol. 46, no. 6, pp. 844-854, 2010.

[23] S.-D. Lee and Y.-C. Fu, "Joint production and shipment lot sizing for a delivery price-based production facility," International Journal of Production Research, vol. 51, no. 20, pp. 6152-6162, 2013.

[24] F. S. Hillier and G. J. Lieberman, Introduction to Operations Research, McGraw-Hill, New York, NY, USA, 2010.

[25] S. L. Grossman, Calculus, Harcourt Brace, New York, NY, USA, 5th edition, 1993.

[26] L. Johnson and D. Montgomery, Operations Research in Production Planning, Scheduling, and Inventory Control, John Wiley \& Sons, New York, NY, USA, 1974. 


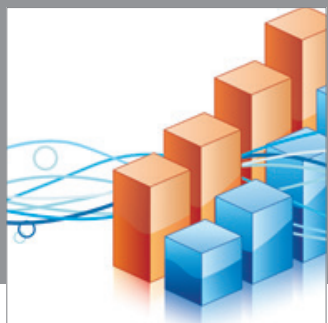

Advances in

Operations Research

mansans

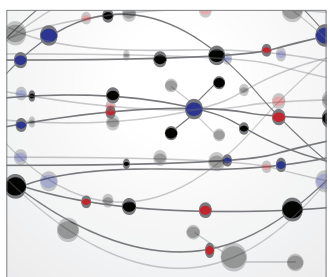

The Scientific World Journal
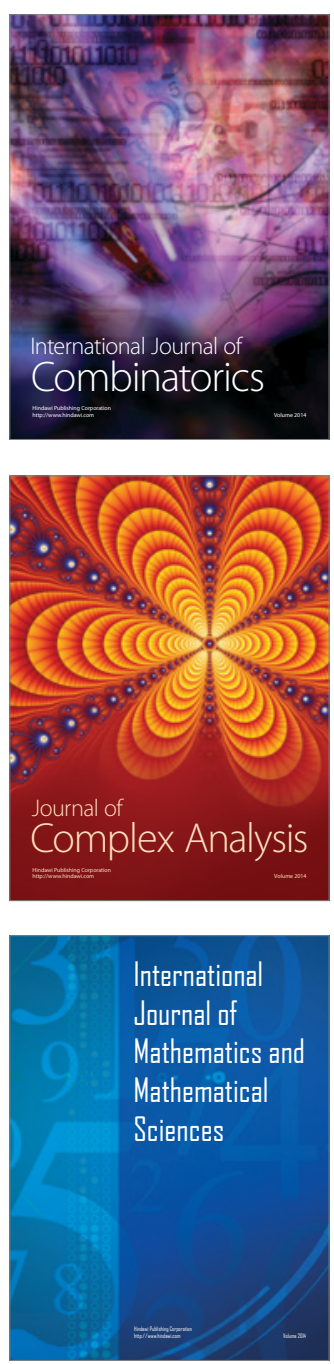
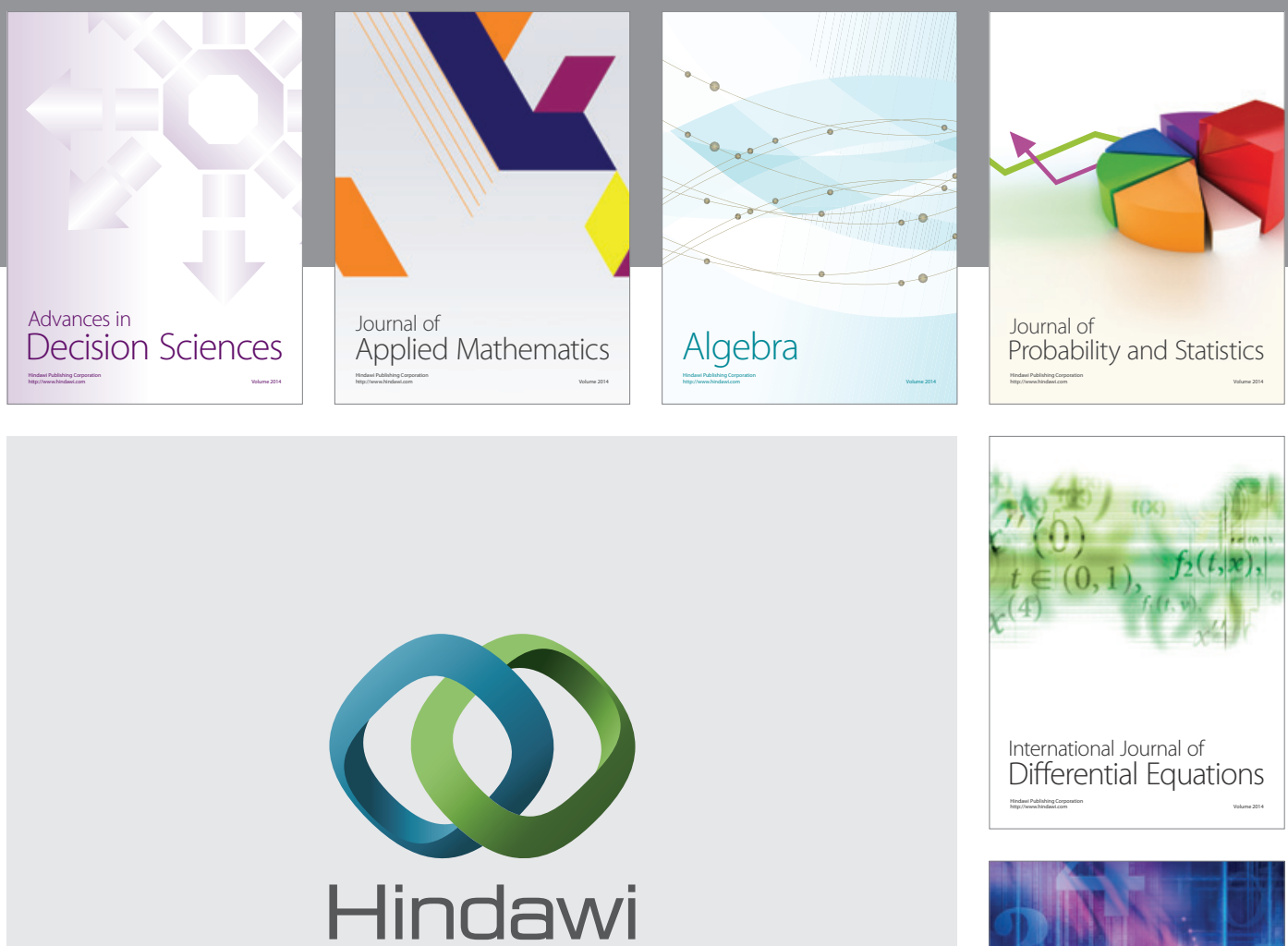

Submit your manuscripts at http://www.hindawi.com
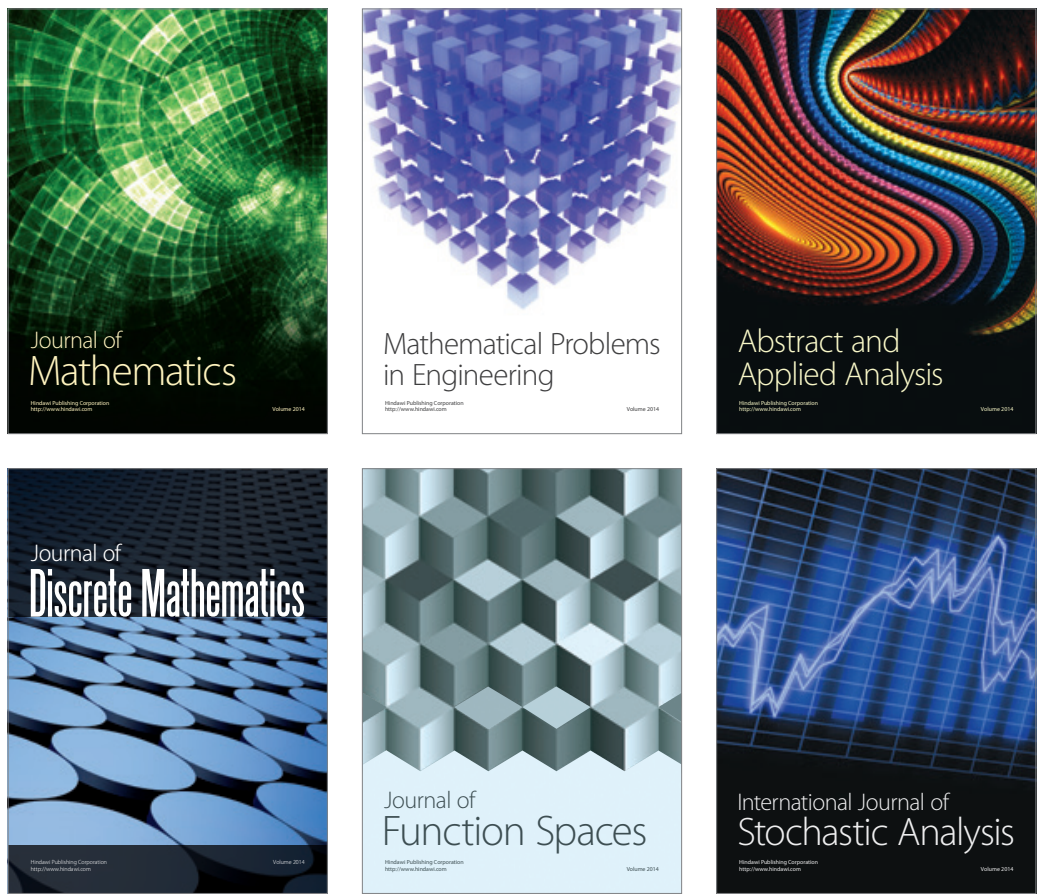

Journal of

Function Spaces

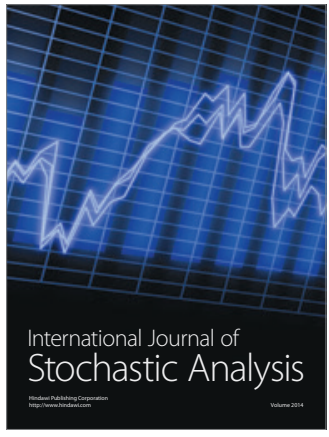

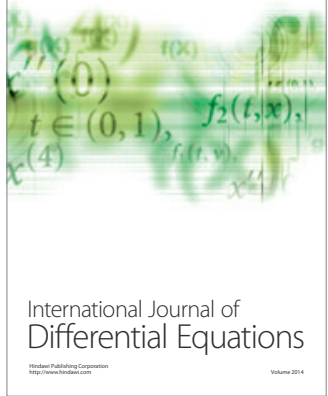
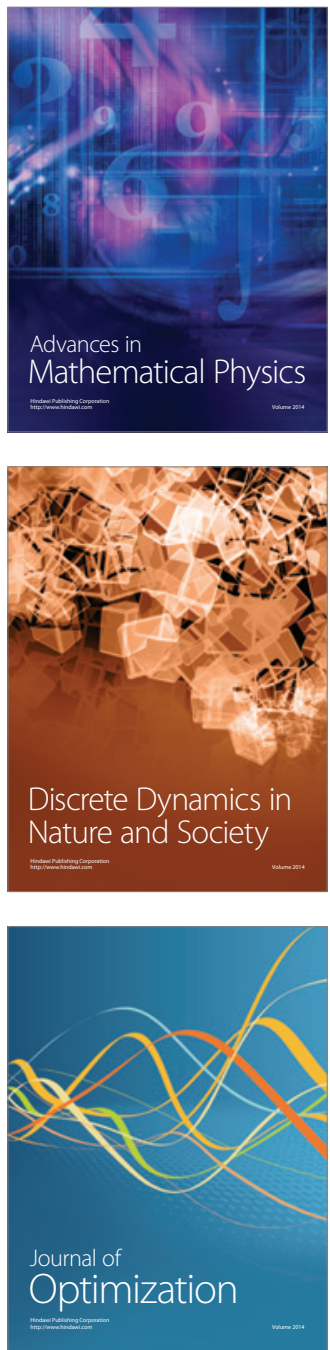